\title{
Dynasore - not just a dynamin inhibitor
}

\author{
Giulio Preta, James G Cronin and I Martin Sheldon ${ }^{*}$
}

\begin{abstract}
Dynamin is a GTPase protein that is essential for membrane fission during clathrin-mediated endocytosis in eukaryotic cells. Dynasore is a GTPase inhibitor that rapidly and reversibly inhibits dynamin activity, which prevents endocytosis. However, comparison between cells treated with dynasore and RNA interference of genes encoding dynamin, reveals evidence that dynasore reduces labile cholesterol in the plasma membrane, and disrupts lipid raft organization, in a dynamin-independent manner. To explore the role of dynamin it is important to use multiple dynamin inhibitors, alongside the use of dynamin mutants and RNA interference targeting genes encoding dynamin. On the other hand, dynasore provides an interesting tool to explore the regulation of cholesterol in plasma membranes.
\end{abstract}

Keywords: Dynasore, GTPase, Dynamin, Endocytosis, Cholesterol, Lipid rafts

\section{Introduction}

Dynamin is an intracellular protein with essential roles in membrane remodelling and fission of clathrin-coated vesicles formed during endocytosis, and vesicles that bud from the trans-Golgi network [1]. In particular, endocytosis is dependent on dynamin for the invagination of plasma membrane to form clathrin-coated pits, and dynamin polymerizes to form a helix around the neck of budding vesicles of plasma membrane leading to membrane fission and generation of free clathrin-coated vesicles (Figure 1A) [2]. Clathrin-mediated endocytosis regulates fundamental cellular processes, including the homeostasis of plasma membrane, receptor turnover, and the uptake of nutrients [3]. On the other hand, many pathogens have evolved to exploit endocytosis to enter eukaryotic cells. As well as linking to the actin cytoskeleton during clathrin-coated vesicle formation, interaction between dynamin and the actin cytoskeleton occurs during the formation of membrane ruffles, lamellapodia, and podosomes [4-6]. In addition, a growing number of dynamin-like proteins have been identified, such as mitochondrial DRP1, which contribute to the fusion and remodelling of intracellular membranes [1,7].

Dynamin is a $100 \mathrm{kDa}$ protein with multiple domains, principally explored by generation of dynamin mutants. Perhaps the most important domain is a large GTPase enzyme essential for membrane fission $[8,9,10]$. In

\footnotetext{
* Correspondence: i.m.sheldon@swansea.ac.uk

Institute of Life Science, College of Medicine, Swansea University, Swansea SA2 8PP, UK
}

(c) 2015 Preta et al.; licensee BioMed Central. This is an Open Access article distributed under the terms of the Creative Commons Attribution License (http://creativecommons.org/licenses/by/4.0), which permits unrestricted use, distribution, and reproduction in any medium, provided the original work is properly credited. The Creative Commons Public Domain Dedication waiver (http://creativecommons.org/publicdomain/zero/1.0/) applies to the data made available in this article, unless otherwise stated. pleckstrin homology domain implicated in membrane binding, a GTPase effector domain essential for selfassembly, and a C-terminal proline-rich domain, which contains several SH3-binding sites [1]. Dynamin partners bind to the proline-rich domain, stimulating dynamin's GTPase activity and targeting dynamin to the plasma membrane [11]. In particular, dynamin is efficiently supplied with GTP by interaction between the dynamin proline-rich domain and nucleoside diphosphate kinases $\mathrm{NM} 23-\mathrm{H} 1 / \mathrm{H} 2$, to trigger membrane fission [12]. Purified dynamin exists as a tetramer [13], which can self-assemble into structures that resemble rings and helices [14]. In Drosophila melanogaster and Caenorhabditis elegans only one dynamin isoform has been identified [15-17]. However, three dynamin-encoding mammalian genes (DNM1, $D N M 2$ and DNM3) have been identified [18,19]. Although the dynamin isoforms have similar functions, including membrane fission during clathrin-mediated endocytosis, dynamin 1 and dynamin 3 are mainly expressed in the brain, whereas dynamin 2 is expressed ubiquitously [1]. Although overexpression of mutants has been used to explore the role of dynamin, even mutations that effectively target the dynamin GTPase, such as dynamin K44A, S45N, T65F and T65A, vary in their potency and the stage at which they inhibit endocytosis $[8,20]$. Whilst the overexpression of dynamin mutants and RNA interference targeting the mammalian dynamin genes has been valuable, progress in understanding the mechanism of action of 


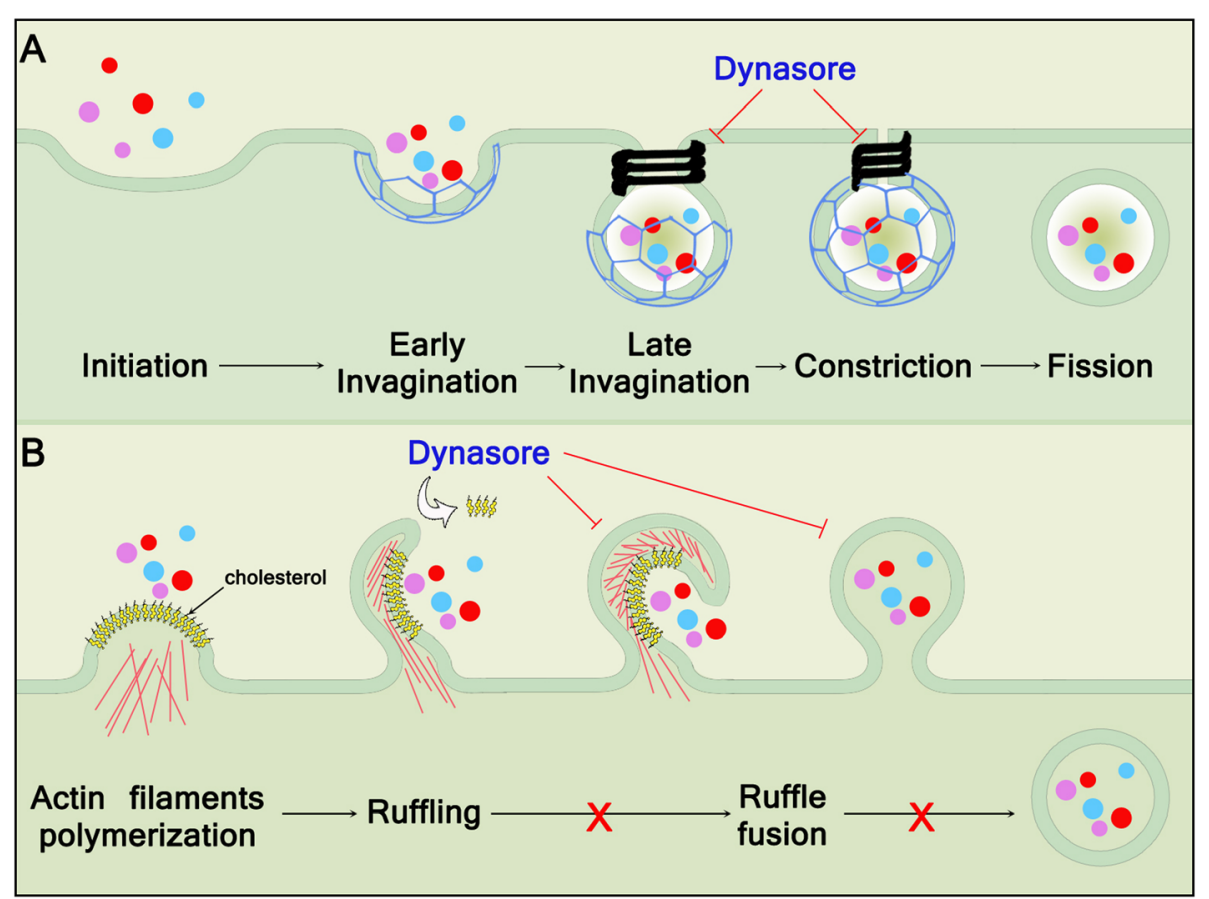

Figure 1 The stages of clathrin-coated vesicle formation. (A) Inititation and early invagination: a clathrin-coated pit is formed and cargo-specific adaptors are selected. Late invagination: further clathrin is recruited and polymerizes in hexagons and pentagons to form the clathrin coat. Constriction: dynamin is recruited to the neck of the forming vesicle where it forms helical structures, and induces membrane scission. Fission: an endocytic vesicle is produced containing cargo molecules. Dynasore inhibits the GTPase activity of dynamin, blocking constriction and fission. (B) The steps leading to macropinocytosis. During the vesicular trafficking process, cellular membranes undergo dynamic morphological changes, in particular at the vesicle generation and fusion steps. Macropinocytosis involves the eruption of membrane ruffles from the cell surface that can fuse with the plasma membrane to engulf surrounding cargo, a process that requires extensive actin mobilization. Macropinosomes then fuse with compartments of the normal endocytic pathway. Dynasore reduces plasma membrane cholesterol, inhibiting mobilization of the cellular membrane.

dynamin has also benefited from the discovery of dynamin inhibitors, including the GTPase inhibitor dynasore [2].

\section{Evolution of dynamin inhibitors}

The first dynamin inhibitors to be identified were ammonium salts, such as myristyl trimethyl ammonium bromides (also known as MiTMAB), and the dimeric tyrphostins [21,22]. Most of the first generation of dynamin inhibitors, and their subsequent derivatives, prevent recruitment of dynamin to membranes. On the other hand, compounds that inhibit ATPases and GTPases, for example dynole 34-2 or dynasore inhibit the activity of dynamin following recruitment of dynamin to plasma membranes [23]. Dynasore was identified by Macia and colleagues by screening $\sim 16,000$ compounds for the ability to inhibit the GTPase activity of dynamin 1 , and evidence for the activity of dynasore included inhibition of endocytosis of the transferrin receptor and low density lipoprotein receptor (LDLR) [2]. A characteristic of dynasore is the non-competitive inhibition of the basal and stimulated rates of GTP hydrolysis, without affecting the affinity for GTP binding or dynamin selfassembly [2]. Within 2 minutes, treatment of cells with dynasore inhibits clathrin-mediated endocytosis, and this effect can be reversed in approximately 20 minutes by removal of the inhibitor (Figure 2A and Table 1) $[2,24]$.

Thus, the discovery of dynasore provided an effective tool to study endocytosis in a range of cell types, and in cells derived from several species, including humans, mice and cattle. However, dynasore also has undesirable properties including the binding of serum proteins, causing the loss of dynamin inhibitory activity [25]. Furthermore, dynasore binds to detergents that are often used for in vitro drug screening, which reduces the potency of the inhibitor. These limitations of dynasore lead to the synthesis of dihydroxyl and trihydroxyl dynasore analogs, called the Dyngo compounds, which have improved potency, reduced cytotoxicity, and reduced detergent binding [26].

\section{Dynasore in the regulation of cholesterol homeostasis: beyond dynamin inhibition}

An emerging role of dynamin is the regulation of cellular cholesterol, and dynasore impacts cholesterol homeostasis. Sixty to $90 \%$ of cellular cholesterol is located in the plasma membrane, and cholesterol forms about half of 


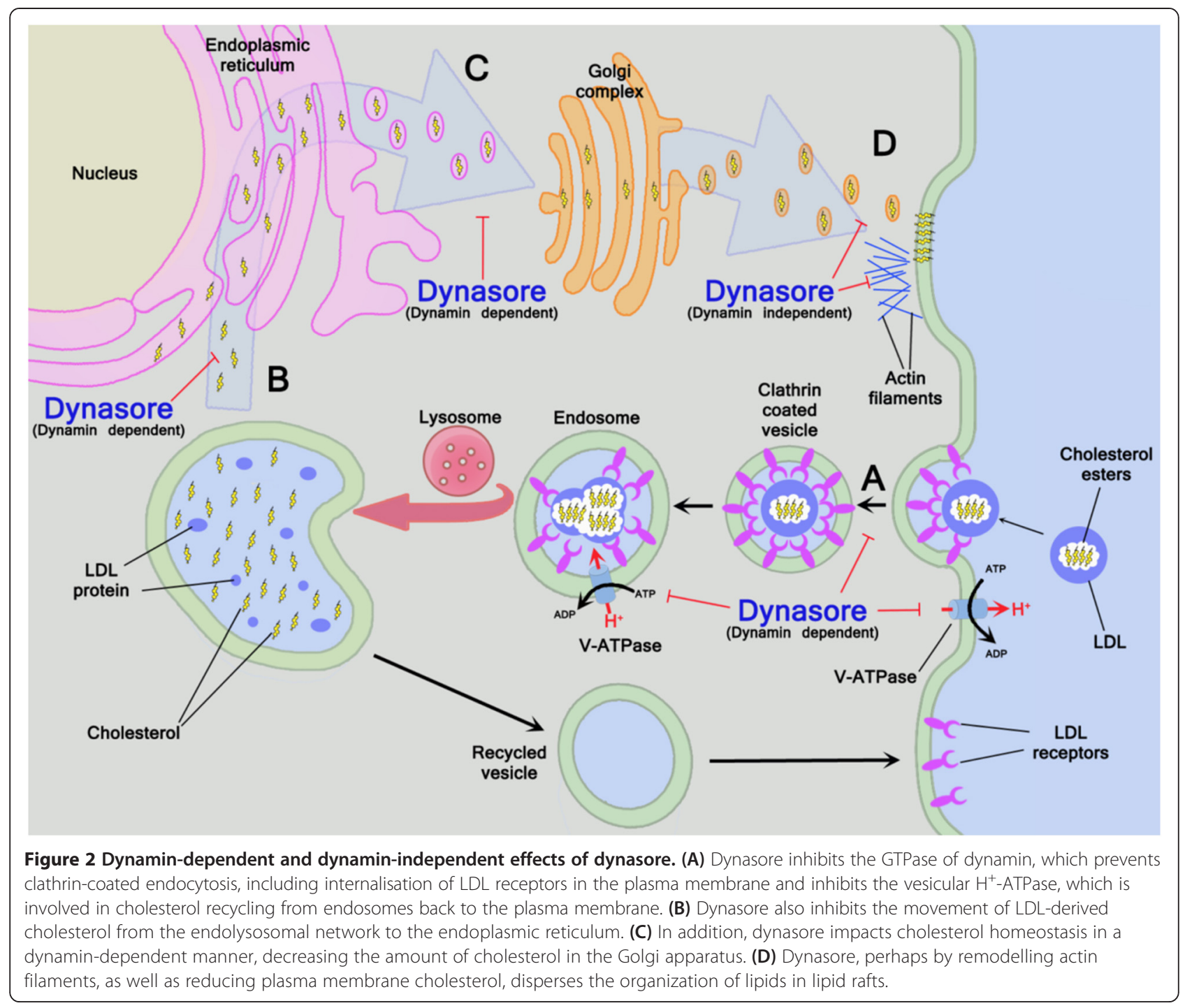

the total plasma membrane lipids [27]. Recent evidence supports a concept for three pools of cholesterol in plasma membranes [28]: a labile pool of cholesterol that is depleted when cells are deprived of cholesterol; cholesterol that is bound to sphyngomyelin and is not labile; and, finally an essential pool of cholesterol that is necessary for cell viability. The amount of cholesterol in the labile, sphyngomyelin-bound, and essential pools may vary between types of cells but is around 16\%, 15\% and $12 \%$ of the plasma membranes of fibroblasts, respectively [28].

Cellular cholesterol homeostasis depends on the balance between sequestration of cholesterol in membranes or cholesterol metabolism, and the uptake of LDLderived cholesterol via endocytosis of the LDLR or cholesterol synthesis via the mevalonate pathway [29]. The LDL-derived cholesterol esters are de-esterified in endolysosomes to release free cholesterol, which transits to the plasma membrane to resupply the pool of labile cholesterol and, once the plasma membrane cholesterol is replete, free cholesterol moves to the endoplasmic reticulum (ER) [28]. Cholesterol synthesis via the mevalonate pathway is controlled by SREBP-2 [30]. When cells have sufficient ER cholesterol, usually $>5 \%$ of ER lipids, SREBP-2 in complex with the escort protein (Scap) is bound to an ER membrane anchor protein Insig. However, when ER cholesterol is $<5 \%$ of ER lipids, the SREBP-2/Scap complex is released from the ER and transported to the Golgi in COPII-coated vesicles. In the Golgi, SREBP-2 is cleaved to release the active form, which enters the nucleus and drives transcription of genes encoding most components of the mevalonate pathway, and for the LDLR. When there is excess cholesterol, or loss of sphingomyelin, plasma membrane cholesterol is delivered to the ER where it is esterified by the ER resident protein ACAT, and cholesterol esters are stored in cytoplasmic droplets. Dynamin also plays a role 
Table 1 Evidence supporting dynamin-dependent and dynamin-independent effects of dynasore

\begin{tabular}{|c|c|c|}
\hline \multicolumn{3}{|l|}{ Dynamin-dependent effects } \\
\hline Effect & Supporting approaches & Reference \\
\hline \multirow[t]{6}{*}{ Clathrin-mediated endocytosis } & Dynasore & {$[1,2,8,20,34]$} \\
\hline & Dynamin mutants & \\
\hline & Triple dynamin knock out & \\
\hline & Dyngo-4a & \\
\hline & Dynamin inhibitor peptide & \\
\hline & siRNA & \\
\hline \multirow[t]{3}{*}{ Accumulation of cholesterol in ER } & Dynasore & {$[31,32]$} \\
\hline & Dynamin mutants & \\
\hline & SiRNA & \\
\hline \multirow[t]{3}{*}{ Golgi vesiculation } & Dynasore & {$[33,53]$} \\
\hline & Dynamin mutants & \\
\hline & SiRNA & \\
\hline \multirow[t]{3}{*}{ Inhibition of V-ATPase activity } & Dynasore & [37] \\
\hline & Dynamin inhibitor peptide & \\
\hline & siRNA & \\
\hline \multicolumn{3}{|l|}{ Dynamin-independent effects } \\
\hline Effect & Supporting approaches & Reference \\
\hline Disruption of lipid rafts & Dynasore differs from dynamin inhibitor peptide and siRNA & {$[24]$} \\
\hline Inhibition of membrane ruffling & Dynasore and Dyngo 4a differ from triple dynamin knock out & [34] \\
\hline Destabilization of F-actin & Dynasore and Dyngo 4a differ from triple dynamin knock out & [34] \\
\hline
\end{tabular}

in cholesterol homeostasis as LDLR internalization depends on endocytosis [2]. Presumably by inhibition of dynamin-dependent endocytosis, dynasore reduces LDL uptake in HeLA cells to $10 \%$ of that of the control [31]. The implication of this observation is that dynasore treatment would then lead to depletion of labile cholesterol in the plasma membrane. However, dynamin also appears to have an additional role in the delivery of free cholesterol from the endolysosomal network to the ER since the use of the K44A mutated form of dynamin, RNA interference targeting dynamin, or dynasore, leads to accumulation of free cholesterol and LDL-derived cholesterol within the late endolysosomal compartment (Figure 2B and Table 1) $[31,32]$. The importance of dynamin in cholesterol homeostasis is further illustrated by the use of the dynamin K44A mutant, as well as transient transfections with dominant negative mutant constructs of dynamin 1 and dynamin 2, which inhibited cholesterol-induced vesiculation of the Golgi (Figure 2C and Table 1) [33]. Taking the above findings together, one inference is that cells deficient in dynamin or treated with dynasore would not initiate mechanisms to increase cellular cholesterol when cholesterol is depleted in different compartments, because the ER contains surplus free cholesterol. In support of this concept, treatment of cells with dynasore reduces LDLR gene expression, although less rapidly than supplying cells with LDL [31].

As well as effects on cellular cholesterol attributable to inhibition of dynamin, recent observations imply that dynasore also influences cholesterol homeostasis in a dynamin-independent manner. As expected, endocytosis of the transferrin receptor was blocked in fibroblast cells that have a triple knockout of DYN1, DYN2 and DYN3, although uptake of dextran, called fluid-phase endocytosis, was not affected [34]. Surprisingly, treatment of the triple knockout fibroblasts with dynasore or Dyngo-4a inhibited fluid-phase endocytosis, implicating dynaminindependent effects of the inhibitors [34]. Furthermore, membrane ruffling was prevented by dynasore or Dyngo-4a but not the triple knockout of dynamin. Membrane ruffles are actin-rich protrusions of the plasma membrane that can be observed on the surface of many cell types, often involved in macropinocytosis (Figure 1B). Macropinocytosis, unlike clathrin-mediated endocytosis, is a dynamin-independent processes [35]. Thus, it appears that dynasore and Dyngo-4a have unexpected offtarget effects. One possibility is an effect on plasma membrane cholesterol because extraction of cholesterol with methyl- $\beta$-cyclodextrin also inhibits the formation of membrane ruffles at the plasma membrane, and inhibits 
the reorganization of filamentous actin at the cell periphery necessary for the formation of membrane ruffles (Figure 2D and Table 1) [36]. A further potential mechanism underlying the dynamin-independent effect of dynasore on cellular cholesterol is related to dynasore inhibition of vacuolar $\mathrm{H}^{+}$-ATPase (V-ATPase) enzymes (Figure 2D and Table 1) [37]. Inhibition of V-ATPase perturbs clathrin-coated vesicle formation, with retention of cholesterol in non-acidified endosomes, and loss of cholesterol from the plasma membrane, and the effect is partially rescued be providing exogenous cholesterol [38]. Reduction of passive cholesterol efflux from HeLA cells and macrophages also provides supporting evidence that dynasore reduces the labile pool of plasma membrane cholesterol [31]. The mechanism for this "off-target" effect is not known, but it is interesting to note that GTPase activity is important for assembly of the COPIIcoated vesicles of liposomes and endoplasmic reticulum $[39,40]$.

\section{Dynasore targets lipid rafts}

Lipid rafts are membrane microdomains that are enriched in cholesterol, sphingomyelin, sphingolipids and phospholipids, and these areas of membrane differ in composition from the surrounding regions of plasma membrane [41,42]. Lipid rafts contribute to the compartmentalization of membranes and the spatiotemporal regulation of cellular signalling. Pathogenic bacteria and viruses also exploit lipid rafts to cause pathology or to gain entry into mammalian cells [43-45]. Microbes not only target the clusters of receptors often concentrated in lipid rafts but also utilize their cholesterol-rich microdomains [46]. In particular, the pore-forming, cholesteroldependent cytolysins, such as Aerolysin, bind to lipid rafts [47]. Some cholesterol-dependent cytolysins bind to cellular receptors that are enriched in lipid rafts; glycosyl phosphatidylinositol-anchored receptors in the case of Aerolysin. However, other cholesterol-dependent cytolysins, such as Perfringolysin O, bind the labile cholesterol in cellular membranes [28]. Cholesterol-dependent cytolysins multimerise in plasma membranes to form pores, leading to osmotic cell death. As might be expected, depletion of cellular cholesterol using methyl- $\beta$-cyclodextrin is protective against the effect of cholesterol-dependent cytolysins [48]. However, dynasore was recently reported to protect HeLA cells and fibroblasts from the toxic effect of two members of the cholesterol-dependent cytolysin family, Pyolysin and Streptolysin-O, as efficiently as methyl- $\beta$-cyclodextrin [24]. Moreover, there was evidence that the effect of dynasore was dynamin-independent because RNA interference targeting to reduce dynamin expression did not protect against Pyolysin. The dynaminindependent effect of dynasore was not only associated with reduced cellular cholesterol but also dispersal of plasma membrane lipid rafts (Figure 2D and Table 1). Similarly, the lipid raft-dependent uptake of the subtilase cytotoxin of Escherichia coli was suppressed by dynasore, but was not influenced by RNA interference targeting dynamin expression [49]. The importance of disruption of lipid rafts is also supported by studies of innate immunity, where cell plasma membrane receptors such as Toll-like receptor 4 (TLR4) and CD14, which bind the pathogenassociated molecule lipopolysaccharide (LPS), are localised to lipid rafts [50]. Indeed, dynasore also reduced the inflammatory cytokine response to LPS in fibroblasts [24]. It would be interesting for future work to explore if dynasore impacts not only labile cholesterol in plasma membranes, but also the sphyngomyelin-bound cholesterol in lipid rafts. This would be particularly important as statin and cyclodextrin molecules only appear to deplete the labile pool of cholesterol in plasma membranes [28].

In addition to changes in plasma membrane cholesterol, the physical properties of cell membranes and the shape of cells may be modulated by interactions between dynasore and actin. Indeed, dynasore destabilizes and remodels F-actin in vitro [51,52]. Dynamin triple knockout cells changed shape following dynasore treatment, providing evidence for a dynamin-independent effects on actin (Figure 2D and Table 1) [34]. Taken together, the inhibition of membrane ruffles and prevention of CDCmediated cytolysis by dynasore [31,24], implies that dynasore actively influences the content and distribution of cholesterol in plasma membranes, and that this is independent of dynamin. Further work is now need to determine the mechanism by which dynasore exerts dynamin-independent effects on mammalian cells.

\section{Conclusion}

Dynasore provides rapid and reversible inhibition of dynamin-dependent endocytosis, which is effective in cells from several species. However, in addition to inhibition of the GTPase of dynamin, dynasore has wider effects on cellular cholesterol, lipid rafts, and actin. The mechanisms associated with these "off-target" effects require further exploration. Understanding how dynasore modulates plasma membrane cholesterol is particularly intriguing as this may uncover novel methods to counter pathogen entry, and reduce the impact of cholesteroldependent cytolysins and other pore-forming toxins on cell viability. However, caution is required when using dynasore to determine the role of dynamin in the biology of cells. Robust evidence for the impact of dynamin likely requires the combined use of several dynamin inhibitors, alongside RNA interference targeting the genes encoding dynamin.

\section{Competing interests}

The authors declare that they have no competing interests. 


\section{Authors' contributions}

GP and IMS wrote the paper, and JGC prepared the figures. All authors read and approved the final manuscript.

\section{Acknowledgement}

Work in the Sheldon laboratory is funded by the U.K. Biotechnology and Biological Sciences Research Council (grant number BB/K006592/1).

\section{Received: 4 February 2015 Accepted: 26 March 2015}

\section{Published online: 10 April 2015}

\section{References}

1. Ferguson SM, De Camilli P. Dynamin, a membrane-remodelling GTPase. Nat Rev Mol Cell Biol. 2012;13:75-88.

2. Macia E, Ehrlich M, Massol R, Boucrot E, Brunner C, Kirchhausen T. Dynasore, a cell-permeable inhibitor of dynamin. Dev Cell. 2006;10:839-50.

3. McMahon HT, Boucrot E. Molecular mechanism and physiological functions of clathrin-mediated endocytosis. Nat Rev Mol Cell Biol. 2011;12:517-33.

4. Orth JD, Krueger EW, Cao H, McNiven MA. The large GTPase dynamin regulates actin comet formation and movement in living cells. Proc Natl Acad Sci U S A. 2002;99:167-72.

5. Ochoa GC, Slepnev VI, Neff L, Ringstad N, Takei K, Daniell L, et al. A functional link between dynamin and the actin cytoskeleton at podosomes. J Cell Biol. 2000;150:377-89.

6. Jones SM, Howell KE, Henley JR, Cao H, McNiven MA. Role of dynamin in the formation of transport vesicles from the trans-Golgi network. Science. 1998;279:573-7.

7. Williams M, Kim K. From membranes to organelles: emerging roles for dynamin-like proteins in diverse cellular processes. Eur J Cell Biol. 2014;93:267-77.

8. Marks B, Stowell MH, Vallis Y, Mills IG, Gibson A, Hopkins CR, et al. GTPase activity of dynamin and resulting conformation change are essential for endocytosis. Nature. 2001;410:231-5.

9. McNiven MA. Dynamin: a molecular motor with pinchase action. Cell. 1998:94:151-4

10. Sever S, Damke H, Schmid SL. Dynamin:GTP controls the formation of constricted coated pits, the rate limiting step in clathrin-mediated endocytosis. J Cell Biol. 2000;150:1137-48.

11. Hinshaw JE. Dynamin and its role in membrane fission. Annu Rev Cell Dev Biol. 2000;16:483-519.

12. Boissan M, Montagnac G, Shen Q, Griparic L, Guitton J, Romao M, et al. Membrane trafficking. Nucleoside diphosphate kinases fuel dynamin superfamily proteins with GTP for membrane remodeling. Science. 2014:344:1510-5.

13. Muhlberg $A B$, Warnock DE, Schmid SL. Domain structure and intramolecular regulation of dynamin GTPase. EMBO J. 1997;16:6676-83.

14. Hinshaw JE, Schmid SL. Dynamin self-assembles into rings suggesting a mechanism for coated vesicle budding. Nature. 1995;374:190-2.

15. Chen MS, Obar RA, Schroeder CC, Austin TW, Poodry CA, Wadsworth SC, et al. Multiple forms of dynamin are encoded by shibire, a Drosophila gene involved in endocytosis. Nature. 1991;351:583-6.

16. van der Bliek AM, Meyerowitz EM. Dynamin-like protein encoded by the Drosophila shibire gene associated with vesicular traffic. Nature. 1991;351:411-4.

17. Clark SG, Shurland DL, Meyerowitz EM, Bargmann Cl, van der Bliek AM. A dynamin GTPase mutation causes a rapid and reversible temperatureinducible locomotion defect in C. elegans. Proc Natl Acad Sci U S A. 1997;94:10438-43.

18. Cao H, Garcia F, McNiven MA. Differential distribution of dynamin isoforms in mammalian cells. Mol Biol Cell. 1998;9:2595-609.

19. Urrutia R, Henley JR, Cook T, McNiven MA. The dynamins: redundant or distinct functions for an expanding family of related GTPases? Proc Natl Acad Sci U S A. 1997;94:377-84.

20. Damke H, Binns DD, Ueda H, Schmid SL, Baba T. Dynamin GTPase domain mutants block endocytic vesicle formation at morphologically distinct stages. Mol Biol Cell. 2001;12:2578-89.

21. Hill TA, Odell LR, Quan A, Abagyan R, Ferguson G, Robinson PJ, et al. Long chain amines and long chain ammonium salts as novel inhibitors of dynamin GTPase activity. Bioorg Med Chem Lett. 2004;14:3275-8.
22. Hill T, Odell LR, Edwards JK, Graham ME, McGeachie AB, Rusak J, et al. Small molecule inhibitors of dynamin I GTPase activity: development of dimeric tyrphostins. J Med Chem. 2005;48:7781-8.

23. Hill TA, Gordon CP, McGeachie AB, Venn-Brown B, Odell LR, Chau N, et al. Inhibition of dynamin mediated endocytosis by the dynoles-synthesis and functional activity of a family of indoles. J Med Chem. 2009;52:3762-73.

24. Preta G, Lotti V, Cronin JG, Sheldon IM. Protective role of the dynamin inhibitor Dynasore against the cholesterol-dependent cytolysin of Trueperella pyogenes. FASEB J. 2015;29:1516-28.

25. Kirchhausen T, Macia E, Pelish HE. Use of dynasore, the small molecule inhibitor of dynamin, in the regulation of endocytosis. Methods Enzymol. 2008;438:77-93.

26. McCluskey A, Daniel JA, Hadzic G, Chau N, Clayton EL, Mariana A, et al. Building a better dynasore: the dyngo compounds potently inhibit dynamin and endocytosis. Traffic. 2013;14:1272-89.

27. Lange $Y$, Swaisgood MH, Ramos BV, Steck TL. Plasma membranes contain half the phospholipid and $90 \%$ of the cholesterol and sphingomyelin in cultured human fibroblasts. J Biol Chem. 1989;264:3786-93.

28. Das A, Brown MS, Anderson DD, Goldstein JL, Radhakrishnan A. Three pools of plasma membrane cholesterol and their relation to cholesterol homeostasis. Elife. 2014;3:e02882.

29. Brown MS, Goldstein JL. A receptor-mediated pathway for cholesterol homeostasis. Science. 1986;232:34-47.

30. Brown MS, Goldstein JL. The SREBP pathway: regulation of cholesterol metabolism by proteolysis of a membrane-bound transcription factor. Cell. 1997;89:331-40

31. Girard E, Paul JL, Fournier N, Beaune P, Johannes L, Lamaze C, et al. The dynamin chemical inhibitor dynasore impairs cholesterol trafficking and sterol-sensitive genes transcription in human HeLa cells and macrophages. PLoS One. 2011;6:e29042.

32. Robinet $\mathrm{P}$, Fradagrada A, Monier MN, Marchetti M, Cogny A, Moatti N, et al. Dynamin is involved in endolysosomal cholesterol delivery to the endoplasmic reticulum: role in cholesterol homeostasis. Traffic. 2006;7:811-23.

33. Grimmer S, Ying M, Walchli S, van Deurs B, Sandvig K. Golgi vesiculation induced by cholesterol occurs by a dynamin- and CPLA2-dependent mechanism. Traffic. 2005;6:144-56.

34. Park RJ, Shen H, Liu L, Liu X, Ferguson SM, De Camilli P. Dynamin triple knockout cells reveal off target effects of commonly used dynamin inhibitors. J Cell Sci. 2013;126:5305-12.

35. Nichols B. Caveosomes and endocytosis of lipid rafts. J Cell Sci. 2003;116:4707-14

36. Grimmer S, van Deurs B, Sandvig K. Membrane ruffling and macropinocytosis in A431 cells require cholesterol. J Cell Sci. 2002;115:2953-62.

37. Sakai H, Moriura Y, Notomi T, Kawawaki J, Ohnishi K, Kuno M. Phospholipase C-dependent $\mathrm{Ca} 2+-$ sensing pathways leading to endocytosis and inhibition of the plasma membrane vacuolar $\mathrm{H}+-$ ATPase in osteoclasts. Am J Physiol Cell Physiol. 2010;299:C570-8.

38. Kozik P, Hodson NA, Sahlender DA, Simecek N, Soromani C, Wu J, et al. A human genome-wide screen for regulators of clathrin-coated vesicle formation reveals an unexpected role for the V-ATPase. Nat Cell Biol. 2013;15:50-60.

39. Antonny B, Madden D, Hamamoto S, Orci L, Schekman R. Dynamics of the COPII coat with GTP and stable analogues. Nat Cell Biol. 2001;3:531-7.

40. Yoshihisa T, Barlowe C, Schekman R. Requirement for a GTPase-activating protein in vesicle budding from the endoplasmic reticulum. Science. 1993;259:1466-8

41. Simons K, van Meer G. Lipid sorting in epithelial cells. Biochemistry. 1988;27:6197-202.

42. Simons K, Ikonen E. Functional rafts in cell membranes. Nature. 1997;387:569-72.

43. Zaas DW, Duncan M, Rae Wright J, Abraham SN. The role of lipid rafts in the pathogenesis of bacterial infections. Biochim Biophys Acta. 2005;1746:305-13.

44. Bavari S, Bosio CM, Wiegand E, Ruthel G, Will AB, Geisbert TW, et al. Lipid raft microdomains: a gateway for compartmentalized trafficking of Ebola and Marburg viruses. J Exp Med. 2002;195:593-602

45. Barman S, Nayak DP. Lipid raft disruption by cholesterol depletion enhances influenza A virus budding from MDCK cells. J Virol. 2007:81:12169-78.

46. Lafont F, van der Goot FG. Bacterial invasion via lipid rafts. Cell Microbiol. 2005;7:613-20.

47. Abrami L, van Der Goot FG. Plasma membrane microdomains act as concentration platforms to facilitate intoxication by aerolysin. J Cell Biol. 1999;147:175-84 
48. Amos MR, Healey GD, Goldstone RJ, Mahan S, Duvel A, Schuberth HJ, et al. Differential endometrial cell sensitivity to a cholesterol-dependent cytolysin links Trueperella pyogenes to uterine disease in cattle. Biol Reprod. 2014;90:54,1-13.

49. Nagasawa S, Ogura K, Tsutsuki H, Saitoh H, Moss J, Iwase $H$, et al. Uptake of Shiga-toxigenic Escherichia coli SubAB by HeLa cells requires an actin- and lipid raft-dependent pathway. Cell Microbiol. 2014;16:1582-601.

50. Triantafilou M, Miyake K, Golenbock DT, Triantafilou K. Mediators of innate immune recognition of bacteria concentrate in lipid rafts and facilitate lipopolysaccharide-induced cell activation. J Cell Sci. 2002;115:2603-11.

51. Mooren OL, Kotova TI, Moore AJ, Schafer DA. Dynamin2 GTPase and cortactin remodel actin filaments. J Biol Chem. 2009;284:23995-4005

52. Yamada H, Abe T, Li SA, Masuoka Y, Isoda M, Watanabe M, et al. Dynasore, a dynamin inhibitor, suppresses lamellipodia formation and cancer cell invasion by destabilizing actin filaments. Biochem Biophys Res Commun. 2009;390:1142-8

53. Weller SG, Capitani M, Cao H, Micaroni M, Luini A, Sallese M, et al. Src kinase regulates the integrity and function of the Golgi apparatus via activation of dynamin 2. Proc Natl Acad Sci U S A. 2010;107:5863-8.

\section{Submit your next manuscript to BioMed Central and take full advantage of:}

- Convenient online submission

- Thorough peer review

- No space constraints or color figure charges

- Immediate publication on acceptance

- Inclusion in PubMed, CAS, Scopus and Google Scholar

- Research which is freely available for redistribution 